\title{
Imaginario profesional: sueños y realidades en enfermería
}

\author{
Araceli Jiménez Mendoza, ${ }^{*}$ Elvira Rodríguez Antinori**
}

\begin{abstract}
RESUMEN
La perspectiva imaginaria de enfermería en los estudiantes de licenciatura, su concepción de la disciplina y su conocimiento acerca del objeto de estudio son ejes sustantivos para la elección de la carrera y el desempeño durante el proceso formativo de su proyecto de vida. La sociedad imagina a las enfermeras como personas que cuidan a los enfermos, que les orientan en el manejo de situaciones relacionadas con la enfermedad, capaces de reconfortarles; sin embargo, en la realidad aún se desconocen algunos aspectos de la naturaleza de su práctica, el ámbito de ejercicio, sus conocimientos y habilidades.
\end{abstract}

Palabras clave: Enfermería, imaginario profesional, cuidado.

\section{Professional imaginary: dreams and realities in nursing}

\begin{abstract}
The imaginary perspective nursing undergraduates, his conception of the discipline and its knowledge about the object of study are substantive axes career choice, performance during the training process of their life project. The nurses imagines society as people who care for the sick, guide them in the management of disease-related situations, able to comfort them, but in reality are still unknown aspects of the nature of their practice, the field exercise, knowledge and skills.
\end{abstract}

Key words: Nursing, professional imaginary, care.

\section{INTRODUCCIÓN}

$\mathrm{L}$ a enfermería moderna surge en el siglo XIX, en Inglaterra, con Florence Nightingale, quien promovió la creación de la Escuela de Enfermería de St. Thomas' Hospital, donde se formaban las enfermeras durante un año; el curso era básicamente práctico y se les evaluaba a través de informes mensuales de conducta y rendimiento, para lo cual tenían que alojarse en el hospital y pagar por su formación; al concluir, podían trabajar donde desearan. Las principales condiciones eran: estar sobria, ser veraz, digna de confianza, ser pura, quieta, honesta, puntual, ordenada y limpia.

Al término de sus estudios, contaban con las habilidades para realizar curaciones, aplicar sanguijuelas, administrar enemas, aplicar vendajes, asear pacientes, asistir en las operaciones, observar características de las secreciones, tomar el pulso, evaluar apetito y sueño; en sí, proporcionar atención a convalecientes, asear camas, realizar limpieza y ventilar las salas de los pacientes. ${ }^{1}$

En México, en la época precortesiana ya existían personas que brindaban atención a los pacientes en los domicilios,

* Maestría en Ciencias de Enfermería, Doctorante de Enfermería, Universidad Nacional de Trujillo, Perú-UNAM.

** Tutora, Universidad Nacional de Trujillo, Perú-UNAM.

Correspondencia: Araceli Jiménez Mendoza. Antiguo Camino a Xochimilco y Viaducto Tlalpan, Col. San Lorenzo Huipulco, 14370, Tlalpan, México, D.F. Tel. 5556 2332. E-mail: ajimenez55070@gmail.com

Este artículo puede ser consultado en versión completa en http://www.medigraphic.com/enfermerianeurologica 
actividad que continuó con la llegada de los españoles y ante la presencia de las epidemias de viruela, cólera y las enfermedades transmisibles. A su vez, se crearon hospitales dirigidos por religiosas, atendidos por mujeres, quienes sólo recibían por sus servicios comida y techo, por considerarse el cuidado un acto de caridad cristiana.

En la época de la Reforma, las órdenes religiosas fueron expulsadas de los hospitales, razón por la cual se integró personal doméstico y mujeres de dudosa conducta para brindar cuidado a los pacientes. En esa época, el empirismo predominaba ante el desempeño académico del personal, ${ }^{2}$ así mismo, el trabajo voluntario por mujeres fue llamado faenas. Durante el porfiriato, los hospitales carecían de personal y para solucionar esto improvisaban, lo cual sin duda se reflejaba en los resultados, por lo que los médicos de esa época propusieron capacitar a las mujeres que les ayudaban, siempre y cuando no fueran analfabetas, lo que originó la imagen de enfermería: "Arte y técnica para ayudar al médico". ${ }^{3}$

Es así como, en 1907, nace el Hospital General Escuela de Enfermería Mexicana, con el fin de formar a las nuevas enfermeras en la atención de los enfermos. En 1911, la EEM se incorporó a la Escuela de Medicina de la Universidad Nacional y a partir de 1945 se convierte en Escuela Nacional de Enfermería y Obstetricia (ENEO). Sin embargo, aún fue dirigida por médicos hasta 1975, cuando asume por primera vez la dirección una enfermera; en ese periodo se adquieren instalaciones propias para la ENEO.

Los planes de estudios de enfermería y los requisitos de ingreso han sido modificados a medida que la profesión ha evolucionado; en sus inicios se requerían tres años de primaria y cubrir estudios de enfermería; posteriormente, primaria completa y cumplir un programa de dos años de carrera; después primaria completa y tres años de carrera. En años recientes, para el nivel técnico universitario se solicitaba secundaria terminada y cubrir tres años de estudios de la carrera y un año de servicio social. A partir de 1968 se imparte la Licenciatura en Enfermería y Obstetricia con cuatro años de carrera y un año de servicio social; cabe aclarar que la universidad mantuvo los dos niveles educativos por varias décadas.

Es importante señalar que el desarrollo de la enfermería mexicana ha tenido una relación estrecha con la consolidación del Sistema de Salud del país desde los años 40; las instituciones fundadas en esa época, como el IMSS, el Hospital Infantil de México, el Instituto Nacional de la Nutrición "Salvador Zubirán", el Instituto Nacional de Cardiología "Ignacio Chávez" tenían de manera sustantiva un enfoque curativo, lo cual impactó en la formación de las enfermeras, a pesar de que en 1950 se incorporó la materia de Salud Pública y de Enfermería Sanitaria. ${ }^{4}$
Cabe destacar que, en el Sector Salud, se ubica al profesional de enfermería como alguien orientado principalmente al cuidado de las personas enfermas, enfoque que requiere ser reorientado hacia nuevos espacios de ejercicio, en donde la enfermedad deje de ser el evento instituido y el problema a solucionar en el estrecho, sufrido y costoso espacio hospitalario, en lugar de ampliar el papel de enfermería hacia la conservación, protección o promoción de la salud y ampliar su saber y sus ámbitos de ejercicio, como son la salud en el trabajo, en los colegios, la salud ambiental nutrida de concepciones ecosóficas, con una perspectiva de mejorar la calidad de vida, con enfoque interdisciplinario, inter y transectorial.

La disciplina de enfermería, si bien ha evolucionado en el contexto de los fenómenos histórico-sociales como otras carreras, aún conserva componentes que la caracterizan como una profesión de bajo status social, lo cual influye en su elección por quienes aspiran a una formación profesional. El estudio del imaginario de los(as) estudiantes de enfermería permite comprender los significados, los objetivos y sus aspiraciones, a partir de los elementos autobiográficos, resultado del análisis de las entrevistas realizadas, como producto de aprendizaje en el seminario de investigación de Metodologías Emergentes.

\section{CONCEPCIONES HACIA EL FUTURO}

La imagen ha sido definida como la "similitud o signo de las cosas, que puede conservarse independientemente de las cosas mismas". Aristóteles planteó que las imágenes "son las cosas sensibles mismas, excepto que no tienen materia", de tal forma que es resultado de la imaginación y la sensación o percepción misma. Luego entonces, la imagen se diferencia de la imaginación por ser esta última la "posibilidad de evocar o producir imágenes, independientemente de la presencia del objeto al cual se refieren".

Bachelard enfoca la imagen o imaginario como "el mundo de los productos concretos: mitos, sueños, creaciones poéticas"; Ricoeur, a su vez, enfatiza en el papel creativo de lo imaginario como "estímulo para el pensamiento conceptual, para pensar más y hablar de otro modo". ${ }^{6}$

Castoriadis, ${ }^{7}$ refiere que si bien la sociedad está conformada por significaciones que le dan sentido a la vida colectiva e individual, lo histórico-social es producto de nuevos significados de imaginarios sociales.

Imaginario social es el conjunto de significaciones por las cuales un colectivo, un grupo, una sociedad, se instituye como tal. ${ }^{8}$

De los intercambios de reflexión interna entre formalización histórica del relato de ficción y la formalización de la ficción del relato histórico surge el tiempo humano o tiempo 
narrado, por lo que la imaginación en el relato histórico es la función de lo imaginario del pasado "real" y el mundo "irreal"; el dilema se centra en hacer evidente el haber sido, sin detrimento de la realidad. El carácter del imaginario se manifiesta a través del pensamiento, proceso esencial en la interpretación, a partir de su valor de huella en el contexto de vida, el entorno social y cultural. ${ }^{9}$

El imaginario profesional se concibe como el conjunto de actitudes, perfiles-tipo, valores, objetivos, funciones y mitologías que se integran al imaginario sociocultural, con una serie de representaciones simbólicos que entretejen una red articulada basada en el pasado y los elementos del presente, de tal forma que en el caso de enfermería el resultado actual es producto de los imaginarios y significaciones generados a partir de su surgimiento.

El proceso de identificación de una profesión es activo y complejo, producto de la configuración y reconfiguración de la identidad individual y colectiva en función del entorno. ${ }^{10}$ Lo esencial se centra en los tres estadios del tiempo pasado, presente y futuro-, condición que conforma su propia explicación y revela una constitución del tiempo que se podría llamar estratificada y una jerarquización de los niveles de temporalización que requiere denominaciones distintas: temporalidad, historicidad, intratemporalidad, ${ }^{11}$ de tal forma que la construcción de la historia surge del análisis de lo histórico y la narración de los entrevistados. ${ }^{12}$

\section{EL PAPEL DE LA UNIVERSIDAD EN LA CONSTRUCCIÓN DE IDENTIDADES}

En el presente trabajo se expone el ejercicio preliminar de las narraciones o testimonios relacionadas con el imaginario profesional tomado de 34 estudiantes de primer grado de la Licenciatura de Enfermería, con el fin de identificar su imaginario profesional en relación con su elección de carrera y rescatar el papel de la universidad en la construcción de la identidad profesional.

Las edades de los estudiantes, al momento de las entrevistas, fluctuaban entre 17 y 20 años. En su mayoría manifestaron convivir con sus padres y ser dependientes económicos, situación característica en la cultura mexicana.

La educación de los padres, maestro o líder ${ }^{13}$ influye en la orientación vocacional de los individuos; de igual manera se habla de que el capital familiar, ${ }^{14}$ le brinda los recursos para que de manera eficiente ocupe un lugar en la sociedad; de esta forma, es relevante considerar la actividad laboral diversa de los padres entre comerciantes, empleados o actividad desconocida; por su parte, las madres, en su mayoría, trabajan en el hogar, lo que permite inferir que la herencia educativa de los progenitores pueda influir en el logro de sus aspiraciones profesionales.
La religión católica ha sido determinante en la organización de los servicios de salud y en la creación de escuelas de enfermería para la formación del personal de salud, por lo que es significativo que la totalidad de los entrevistados manifestaran profesar esta religión, aspecto que puede influir en su concepción profesional y papel social.

La filosofía reflexiva de sí mismo busca identificar el sentido de vida y la interpretación de los símbolos que la constituyen, como ejercicio fenomenológico, por lo que la construcción de la subjetividad permite rescatar al individuo en proceso de profesionalización a través de su propia narración, interpretar su imaginario y su identidad profesional. ${ }^{15}$ El proceso de identidad tiene un respaldo cultural, es complejo, de tal forma que el individuo asume su identidad como resultado de la interacción social y no sólo como proceso individual; de esta forma, participa en la cultura que le dio nombre y lugar en la sociedad, resultado de la categorización y juicio.

El proceso de construcción de la identidad no siempre es exitoso, ${ }^{16}$ porque la experiencia de vida, a través de la interacción con otras personas, influye en la decisión final de cada profesionista.

\section{INSERCIÓN SOCIAL}

En la sociedad, los individuos, al tener derechos y obligaciones adquieren un sentido de pertenencia, así como capacidad para influir y participar en el entorno social de su comunidad, lo mismo que para disfrutar las oportunidades fundamentales de la vida; en síntesis: ejercer su ciudadanía. Así se logra la inserción es un estado definido por la cantidad y calidad de participación de la persona en su entorno en general.

Los estudiantes encuestados expresaron haber sido "rechazados para estudiar carreras como medicina, educación física, química en alimentos, comunicación, turismo y sociología, en las principales instituciones públicas de educación superior en el Distrito Federal, la Universidad Nacional Autónoma de México (UNAM), el Instituto Politécnico Nacional (IPN) y la Universidad Autónoma Metropolitana (UAM)".

Cabe aclarar que estas instituciones, al tener sobredemanda de aspirantes a determinadas carreras, otorgan la posibilidad de elegir una segunda opción, con el fin de que accedan a estudios superiores, ya que representa para muchos una oportunidad de probable movilidad social. ${ }^{17}$

En los casos en que eligieron la carrera de enfermería desde la primera opción, los estudiantes manifestaron haber tenido conocimiento de la profesión, a partir de "enfrentarse a la enfermedad, ya sea personalmente o con algún familiar, por lo que tuvieron la oportunidad de 
acercarse a las áreas hospitalarias y tratar al personal de enfermería”, experiencia que les proporcionó mejores elementos para decidir.

Es importante destacar que, en otros casos, eligieron la carrera como estrategia para que al término estudien la carrera deseada o tan sólo para incorporarse al sistema escolar universitario y posteriormente solicitar el cambio de carrera; sin embargo, en la actualidad, en muchos casos, se ven frustradas sus intenciones porque la UNAM tiene criterios definidos de ingreso para las carreras con mayor demanda.

\section{¿QUIÉN SOY? ¿POR QUÉ DESEO SER PROFESIONISTA?}

La identidad está constituida por raíces ontológicas que dan respuesta a ¿quién soy?, y a su vez da cuenta de las implicaciones sociales, económicas, políticas, culturales y religiosas que han permeado en la esencia de la persona, a través del proceso de socialización. ${ }^{18}$

Los estudiantes que aseguran tener el "deseo de ser profesionistas de Enfermería" se cuestionan con frecuencia qué quieren ejercer como especialidad en Enfermería, por lo que contar con estudios de postgrado les permite inferir que representará para ellos mayor estatus.

El deseo de estudiar otra carrera es común en muchos, por lo que sueñan con estudiar otra carrera y permanecer en Enfermería, "terminar los estudios de Enfermería e ingresar a la carrera de medicina, química, turismo, sociología..." Esta situación los mantiene en un estado de incertidumbre, carentes de proyecto de vida definido. En relación con las carreras mencionadas, medicina tiene una trayectoria ancestral de reconocimiento social.

En otros casos, los jóvenes manifiestan que ya no quieren cambiar de carrera y se visualizan titulados, reconocidos socialmente y deseosos de brindar sus servicios, desarrollándose más como personas que como profesionistas. Sin duda, la esperanza de lograrlo les impulsa para replantear su proyecto de vida.

Durante la entrevista, se les preguntó si era un capricho o una obsesión estudiar medicina, química, comunicación, turismo, debido a que en algunos casos habían solicitado dichas carreras como primera opción, por lo que muchos expresaron "sentir coraje ya que otros lo lograron"; sin embargo, también manifestaron su decisión de continuar en Enfermería con el objeto de conseguir trabajo al finalizar la carrera y con recursos propios sustentar los estudios deseados. En un caso, una chica afirmó: "Mi familia influyó en la decisión; ellos me inculcaron la idea de estudiar Enfermería, debido a que mis hermanas también estudiaron lo mismo; así comencé estudios en el Instituto Nacional de Enfer- medades Respiratorias INER, y posteriormente, por pase reglamentario, elegí Enfermería como primera opción; tome la decisión por el salario y el desarrollo profesional." La familia muchas veces interviene en la elección de carrera, sobre todo en las estudiantes del sexo femenino; tal es el caso de una estudiante que a pesar de no terminar aún la carrera expresa tener un gran compromiso por una promesa hecha a su mamá, situación que refleja que la profesión de Enfermería está considerada socialmente para mujeres, de condición socioeconómica baja y, por otra parte, que las madres influyen primordialmente en la orientación vocacional de los hijos. ${ }^{19}$

\section{¿QUÉ ES SER PROFESIONAL?, Y ¿QUÉ SIGNIFICA SER PROFESIONISTA?}

Profesionista es la persona que presta un servicio y a cambio recibe una retribución económica (salario), y que cuenta con una formación académica sustentada en valores, con capacidad para ejercer con compromiso social y autonomía. ${ }^{20}$

Las profesiones han surgido como respuesta a las necesidades sociales; existen aquéllas con una historia ancestral y otras de nueva creación; por consecuencia, unas tienen mejor posicionamiento social, lo cual se ve reflejado en el reconocimiento, demanda laboral, estatus económico y sentido de pertenencia de sus integrantes al gremio profesional. La profesión de Enfermería ha visto afectada su imagen social, por tener como objeto de estudio "el cuidado del cuerpo, lo cual es considerado una práctica indeseable"; ${ }^{21}$ asimismo, al caracterizarse como una tarea femenina, enfocada a la enfermedad, confronta lo imaginado con la realidad, transformándose en frustración, desilusión e inclusive en obstinación al intentar crear una fantasía llamada "sueño". La elección de carrera puede orientar al individuo a concebir un proyecto de vida a corto y mediano plazo, lo cual no es un proceso simple ya que intervienen otros factores; aun así, las respuestas de los estudiantes oscilaron entre "difícil visualizarme como profesionista" a "no me proyecto como profesionista, sólo quiero ayudar a la gente y curar a mí mamá porque está enferma del corazón”. Esto significa que los conocimientos que adquiere le son útiles y los puede aplicar en situaciones familiares; sin embargo, la palabra "ayudar" representa a un colaborador que no asume un liderazgo profesional.

En relación con la pregunta: ¿Cómo se visualizan a 5 y 10 años?, algunos contestan: "Quiero terminar los estudios de Licenciatura en Enfermería y trabajar”; “deseo tener un buen trabajo"; "quiero hacer una especialidad y un doctorado". En otros casos afirman: "Me veo como una enfermera exitosa, reconocida y orgullosa de poder brindar mis servicios."(...) "Me visualizo como alguien útil, que 
ayuda con su conocimiento y experiencia a las personas." En este grupo, lograr el profesionalismo es un medio para trabajar, servir a la sociedad y a través de ello obtener el reconocimiento social.

La formación profesional implica conocimientos, técnicas, habilidades, valores y otros elementos como la creatividad e iniciativa, lo que en su conjunto permite al individuo que no sólo aplique conocimientos y solucione problemas referentes a su práctica, sino también que se convierta en un agente de cambio; de tal manera, también hay quien piensa "En 10 años escribiré un libro de Enfermería con un enfoque socialista", "la profesión es una forma de honrar a los seres que he perdido", "quiero ser buena enfermera, siempre actualizada y nunca olvidar que estaré tratando con seres humanos", "trabajaré con una gran responsabilidad al prestar un servicio a la sociedad, poniendo en alto la institución a la que pertenezco”.

\section{ESTRATEGIAS DE ELECCIÓN VOCACIONAL}

La elección de carrera implica intereses que motivan la conducta vocacional del sujeto; ${ }^{22}$ las motivaciones pueden ser intrínsecas y extrínsecas; ${ }^{23}$ plantean que hay mayor factibilidad de éxito profesional ante una motivación intrínseca. La elección vocacional de los estudiantes varones entrevistados ha sido, casi siempre, a partir de que por cuenta propia fueron hospitalizados y en carne propia pudieron apreciar "la capacidad y atención de los enfermeros". A más de uno, como varón, le llamó la atención el desempeño de éstos, pues desconocían la inserción de hombres en una carrera identificada como femenina.

En una estudiante, su elección se dio "posteriormente a mi embarazo y al observar el desempeño profesional de las enfermeras", por lo que distinguió entre ser profesionista y profesional, pues la diferencia radica en que la primera se refiere a una persona que sustenta un título universitario avalado por la adquisición de conocimientos especializados, habilidades y destrezas; mientras la segunda, se refiere a quien desarrolla una actividad con sentido de responsabilidad, honestidad, empatía, proactividad y creatividad, con el fin de brindar un servicio de calidad.

Otra estudiante, después de haber sido rechazada dos veces de medicina, comentó: "Me enteré de la existencia de la Escuela de Enfermería y decidí incorporarme a una carrera del área de salud."

Por desconocimiento, hay quienes suponen que la carrera de Enfermería implica menor esfuerzo; en tal forma, una joven dijo haber elegido enfermería "por ser más fácil”, ya que "en el bachillerato bajó mi rendimiento, abandoné los estudios pero mis padres me dieron una segunda oportunidad para seguir estudiando”.
En otro caso, al descubrirse a sí misma ante una situación adversa, le permitió definir su orientación vocacional, al reconocer sus habilidades: "El fallecimiento de mi hermano me permitió descubrir que me gusta cuidar y ayudar a la gente; posteriormente lo reafirmé al atender a una tía hasta que murió.”

El antecedente de "hermanas enfermeras y posteriormente trabajar seis años como auxiliar de Enfermería" motivó a otra estudiante lo suficiente para elegir la carrera, con una amplia idea de que la profesión le brindará los satisfactores económicos y de desarrollo.

La influencia de los padres es, en muchos casos, determinante, ya que gracias a la insistencia paterna, según otra estudiante, "hice exámenes para ingresar al IPN y a la UNAM, hasta que finalmente logré un lugar en Enfermería, meta que espero cumplir para responder a los deseos de mis padres".

\section{ATESTACIÓN}

La atestación es testimonio, declaración de la creencia de una persona para asumir una responsabilidad, en donde se mantiene una relación de continuidad y de pertenencia a un presente del que se es consciente, con una pretensión de verdad basada en el pasado. ${ }^{24-26}$

La proyección de los jóvenes a cinco años la manifiestan desde diferentes perspectivas como "trabajando como enfermero en un hospital y a su vez estudiando una especialidad o ya estudiando la carrera de Medicina"; "titulada y comenzando una especialidad"; "terminar de estudiar con buenas calificaciones y posteriormente insertarme laboralmente en un hospital”; "con la carrera de Enfermería poder pagarme la carrera de Medicina”. Estas opiniones manifiestan el deseo general de concluir los estudios y en algunos estudiantes considerar a Enfermería como un medio para continuar con su imaginario profesional.

La creatividad y la innovación son evidencia del profesional, por lo que incluso desde los primeros semestres algunos alumnos manifiestan proyectos a mediano y largo plazo, como "poner una agencia de enfermeras y prestar un buen servicio"; "ayudar y estar cerca de la gente”. Otra alumna afirma: "En esta carrera hay mucho quehacer, pienso dedicarme a la investigación y prestar un buen servicio como enfermera", lo que representa asumir el compromiso de terminar la carrera y ampliar el rol profesional hacia la práctica libre e independiente, con el objetivo de brindar un servicio de calidad.

\section{INFLUENCIAS ANTE LA ELECCIÓN}

Los factores que influyen en la elección de una carrera han sido analizados desde diversas dimensiones; la eco- 
nómica se refiere a los costos, expectativas de ingresos y a la perspectiva de empleo, de tal forma que más de uno piensa "echarle ganas en la universidad y buscar un buen trabajo fuera del país para ganar un buen sueldo y ser buen ejemplo para mis hermanos" e identifican "como beneficio el amplio campo de trabajo, el desarrollo profesional, el salario y la posibilidad de especializarse" en una carrera con tan poca demanda de estudio.

La psicología reconoce que el gusto es un factor determinante en la elección de carrera, de tal forma que: "mi gusto por la carrera y la elección es a través de la influencia de un doctor"; "me observo sola en la elección de carrera y las gestiones que pienso hacer para el cambio de carrera". Como vemos, la elección bien puede ser por consecuencia de un proceso individual o por influencia de alguien.

La sociología se enfoca en el background social como factor determinante para la elección, de tal forma que influyen el nivel educativo de los padres o de los familiares cercanos y el reconocimiento social ${ }^{27}$ (Salas y Martin-Cobos, 2006): "Me interesó ser médico debido al contacto en la asistencia prehospitalaria, facilitado por mi abuelo"; "al parecer, la elección sólo pondera mi propio beneficio"; "el apoyo familiar y de la UNAM me inspiran para concluir la carrera y la especialidad a futuro"; "...quiero darles un buen ejemplo y satisfacción a mis padres e hijos"; "de la carrera pretendo obtener éxito y realización profesional".

\section{PRESENTE Y FUTURO DEL IMAGINARIO}

El deseo de expresar los significados, los objetivos y las preocupaciones para reconocer a la Enfermería en el siglo XXI es una necesidad reconocida por las (os) agremiadas (os) a Enfermería.

Es fundamental tener conocimiento del imaginario profesional de Enfermería desde la perspectiva de sus integrantes, a partir de su formación inicial con la intención de generar propuestas de difusión y análisis de la disciplina, que consideren analizar su imagen en diferentes ámbitos y replantearla, a fin de fortalecer la identidad de las nuevas generaciones.

Algunos autores coinciden en que la imagen profesional de Enfermería del siglo XXI debe ser replanteada a partir de su objeto de estudio, el perfil profesional y desarrollar conductas propositivas e innovadoras, encaminadas a la superación profesional, al empoderamiento y a mejorar los servicios de salud para elevar la calidad de vida de la sociedad.

\section{CONSIDERACIONES FINALES}

Sin duda, los resultados preliminares obtenidos en el primer acercamiento del estudio revelan la concepción que al ingre- so tienen los estudiantes de Enfermería, sobre el significado de ser enfermera(o), sobre el imaginario profesional, así como las razones de su presencia en la ENEO.

En este sentido, se observa pobreza en el conocimiento de la profesión; tal parece que esta carrera está ausente, de no ser porque algunos sujetos del estudio que tuvieron una aproximación al fenómeno disciplinario, no hubieran considerado la opción de carrera viable.

Así mismo, puede desprenderse la idea según la cual la sociedad desconoce la importancia de la profesión de Enfermería, porque la enfermera, está atrapada en la invisibilidad de su práctica, hospitalaria curativa, tecnocrática, deshumanizada y dependiente de otros, no pudiendo lograr impacto en quienes cuida y, consecuentemente, aún no obtiene el reconocimiento social al que aspira.

Por ello, es importante continuar estudiando el imaginario profesional desde el abordaje cualitativo con las herramientas metodológicas de las diferentes corrientes del pensamiento para profundizar en la interpretación de los imaginarios y representaciones, tanto en la sociedad, como en estudiantes y egresados del mercado laboral.

Los profesionales de Enfermería tenemos la expectativa de que, a partir del estudio del imaginario profesional y sus representaciones, se generen propuestas de un nuevo papel de la enfermería mexicana, con compromiso social, autonomía, competencia técnica, científica y humana, en un mercado laboral cada vez más amplio e innovador, basado en la promoción de la salud para la calidad de vida, a fin de proyectar una nueva imagen social.

Por otro lado, en la actualidad algunas de las nuevas generaciones se encuentran en un proceso de rescate de ellos mismos y de reconocimiento de la carrera de Enfermería como una alternativa profesional con mucho futuro.

\section{REFERENCIAS}

1. Casasa, P. Una visión antropológica de la enfermería. en México. México, UNAM- Miguel Ángel Porrúa, 2009.

2. Chacón, L. Conceptualización de Enfermería. en Revista de Enfermería del Instituto Mexicano del Seguro Social. México. 1994 Vol. 6, No. 1, enero-diciembre p. 39-40.

3. Ibídem

4. Pérez C., Castañeda M. 100 años de la ENEO 1907-2006.Libro Electrónico México, ENEO UNAM 2006.

5. Abbagnon, N. Diccionario de Filosofía. 2008. $4^{\mathrm{a}}$ ed. Fondo de Cultura Económica. México.

6. Ibídem

7. Castoriadis, en Falcón, Mabel Inés Desarrollo de Nuevas Significaciones en el Espacio Escolar, el Imaginario Escolar Instituyente en la Escuela, 2002. Julio-Diciembre, No. 024, UDUAL, Distrito Federal, México, (p.32) p. p. 31-36

8. Castoriadis, C. La institución imaginaria de la sociedad, 1983. Tusquets, Barcelona.

9. Ricoeur, P. III Tiempo y narración, el tiempo narrado. Siglo XXI editores. México. 2003. 
10. Hurtado JM. Sobre la construcción de la identidad en México. Nómadas 2003.Julio-Diciembre. No. 11. Universidad Complutense de Madrid. 2004. Madrid España.

11. Ricoeur, P. III Tiempo y narración. El tiempo narrado. $3^{\mathrm{a}}$ ed. Ed. SXXI, 2003. Argentina, p. 723.

12. De Certau M. La escritura de la historia. México: Universidad Iberoamericana; 1993. pp67-118.

13. Barroso M. La experiencia de ser familia. 2a. Ed. Pomaire; 1997.

14. Casillas, M, Chain, R, y Jacóme,N. El origen social de los estudiantes y trayectoria estudiantiles en la Universidad Veracruzana. Revista de la Educación Superior. 2007. abril-junio, año/vol. XXXVI (2) num.142 ANUIES. D.F. México, 9-29.

15. Gewehr, A. Identidad profesional y trayectoria en la universidad. Profesorado. Revista de currículum y formación de profesorado, 2001. año/ vol. 5, No. 02. Universidad de Granada. Granada, España.

16. Castells M. La era de la información. Vols. 1-3. Madrid, España: Alianza; 1997.

17. Rivas, R. Logro educacional en jóvenes y clase social de origen: El caso del Gran Concepción. Última década. Julio, número 028, Centro de Estudios Sociales CIDPA. Villa del Mar, 2008. Chile pp. 123-142.

18. Abad, J. "El individuo y sociedad: la construcción de la identidad personal”. En M.A. García de León y otros, Sociología de la Educación. Barcelona: Barcanova; 1993.
19. Salas, V.M y Martín-Cobos P. M. La demanda de educación superior: un análisis microeconómico con datos de corte transversal. Universidad de Granada. Revista de Educación, España. 2006 pp. 637-660.

20. Fernández J. Elementos que consolidad al concepto de profesión. Notas para su reflexión. Revista Electrónica de Investigación Educativa. 2001; 3 (1). [Consultado el 12 de Mayo 2008]. Disponible en URL: http://redie. uabc.mx/contenido/vol3no2/contenido-fernandez.pdf.

21. Colliere, M. Promover la vida. Madrid: McGraw-Hill; 1993.

22. Rivas, R. op. cit.

23. García, G y Organista, J. Motivación y expectativas para ingresar a la carrera de profesor de educación primaria. Un estudio de tres generaciones de estudiantes normalistas mexicanos de primer ingreso. Revista Electrónica de Investigación Educativa. Año/vol 8. Número 002. UABC. Ensenada, B.C. Revista Electrónica de Investigación Educativa. 2006. Año/vol. 8. Número 002.

24. Ricoeur, P. Sí mismo como otro, Madrid: Siglo XXI Editores; 1996.

25. Ricoeur, P. La lectura del tiempo pasado: Memoria y olvido, Madrid: Arrecife; 1999.

26. Ricoeur, P.La mémoire, l'histoire, l'oublie, París, Seuil. Traducción al Castellano: La memoria, la historia, el olvido, Buenos Aires: Fondo de Cultura Económica; 2004.

27. Salas, M y Martin-Cobos, M. La demanda de educación superior: un análisis microeconómico con datos de corte transversal. Revista de Educación. 2006. Número 339. pp 637-660. 